\title{
Chiral phase structure of QCD with many flavors
}

\author{
H. Gies ${ }^{1, a}$, J. Jaeckel ${ }^{2, b}$ \\ ${ }^{1}$ Institut für theoretische Physik, Philosophenweg 16, 69120 Heidelberg, Germany \\ 2 Deutsches Elektronen Synchrotron, Notkestraße 85, 22607 Hamburg, Germany
}

Received: 12 August 2005 / Revised version: 12 December 2005 /

Published online: 17 March 2006 - (C) Springer-Verlag / Società Italiana di Fisica 2006

\begin{abstract}
We investigate QCD with a large number of massless flavors with the aid of renormalization group flow equations. We determine the critical number of flavors separating the phases with and without chiral symmetry breaking in $\mathrm{SU}\left(N_{\mathrm{c}}\right)$ gauge theory with many fermion flavors. Our analysis includes all possible fermionic interaction channels in the pointlike four-fermion limit. Constraints from gauge invariance are resolved explicitly and regulator-scheme dependencies are studied. Our findings confirm the existence of an $N_{\mathrm{f}}$ window where the system is asymptotically free in the ultraviolet, but remains massless and chirally invariant on all scales, approaching a conformal fixed point in the infrared. Our prediction for the critical number of flavors of the zero-temperature chiral phase transition in $\mathrm{SU}(3)$ is $N_{\mathrm{f}}^{\text {cr }}=10.0 \pm$ 0.29 (fermion) ${ }_{-0.63}^{+1.55}$ (gluon), with the errors arising from approximations in the fermionic and gluonic sectors, respectively.
\end{abstract}

PACS. 11.10.Hi; 11.15.Tk; 11.30.Rd

\section{Introduction}

In order to investigate the dynamics of strongly interacting gauge systems, it has often been a successful strategy to deform the desired system into a more accessible one. For instance, the approximation of a continuum gauge theory by a finite lattice gauge theory can be viewed as such a deformation. In the same spirit, the addition of more symmetries as in supersymmetric versions of gauge theories represents such a more tractable deformation. In the present work, we consider the deformation of $\mathrm{SU}\left(N_{\mathrm{c}}\right)$ gauge theories by many massless fermion flavors. Since light fermions have the perturbative tendency to screen long-range forces, a large fermion flavor number $N_{\mathrm{f}}$ has the potential to move the system towards a weaker gauge interaction, for which analytic tools are more powerful.

The relevance of fermionic screening at large $N_{\mathrm{f}}$ is ultimately observable for $N_{\mathrm{f}}>N_{\mathrm{f}}^{\text {a.f. }}:=\frac{11}{2} N_{\mathrm{c}}$ where asymptotic freedom is lost. But even for smaller $N_{\mathrm{f}}$, fermionic screening is first signaled by the second $\beta$ function coefficient of the gauge coupling, reversing its sign for $N_{\mathrm{f}}>$ $\frac{34 N_{\mathrm{c}}^{3}}{13 \mathrm{~N}_{\mathrm{c}}^{2}-3}$. This sign change induces a second zero of the perturbative $\beta$ function, implying an infrared (IR) attractive fixed point of the gauge coupling $\alpha_{*}>0$. As was first argued in [1], the fixed-point value $\alpha_{*}$ is small for $N_{\mathrm{f}} \lesssim N_{\mathrm{f}}^{\text {a.f. }}$, supporting the possibility of a perturbative analysis. If this

\footnotetext{
a e-mail: h.gies@thphys.uni-heidelberg.de

b e-mail: jjaeckel@mail.desy.de
}

fixed point persists on all scales, the system approaches a conformally invariant limit in the deep IR, keeping massless quark and gluon excitations in the spectrum. This scenario can collapse owing to non-perturbative phenomena such as the spontaneous break-down of chiral symmetry. The latter renders the fermions massive, implying their decoupling at low scales. Fermionic screening properties are thus switched off in the deep IR, and the system is characterized by strongly coupled glue and the Goldstone bosons of chiral symmetry breaking $(\chi \mathrm{SB})$.

Since $\chi \mathrm{SB}$ is triggered by the strength of the gauge interactions which in turn depends on the amount of fermionic screening, we expect the conformal scenario to hold for large $N_{\mathrm{f}}$ sufficiently close to $N_{\mathrm{f}}^{\text {a.f. }}$. The broken phase is supposed to occur for smaller $N_{\mathrm{f}}$ with a critical $N_{\mathrm{f}}^{\text {cr }}$ separating the two phases. In fact, evidence for this phase structure has been collected from various methods, including (improved) ladder truncated Dyson-Schwinger equations [2-4], lattice QCD [5], anomaly-induced effective potentials [6], and instanton models [7], with estimates for the critical number of flavors ranging from $N_{\mathrm{f}}^{\mathrm{cr}} \simeq 5[8]$ to $N_{\mathrm{f}}^{\text {cr }} \simeq 12$ for $\mathrm{SU}(3)$ gauge theory.

An analysis of the quark-scattering amplitude using the functional methods $[2,3]$ particularly reveals that the nature of the phase transition, though continuous, is not conventionally second order. ${ }^{1}$ This is most prominently visible

\footnotetext{
1 Owing to the different conformal properties of the system on each side, the transition is often referred to as a conformal phase transition [2].
} 
in the fact that there appear to be no light scalar states in terms of which an effective theory could be constructed on the symmetric side $\left(N_{\mathrm{f}} \gtrsim N_{\mathrm{f}}^{\text {cr }}\right)$ of the phase transition $[2,3,9]$. Any attempt at constructing a GinzburgLandau type of effective potential for the chiral order parameter hence produces discontinuities in the potential parameters. On the other hand, the order parameter in the form of the chiral condensate, i.e., the minimum of this would-be potential, changes continuously across the phase transition.

In this work, we analyze the phase structure of manyflavor QCD with the aid of the functional renormalization group (RG). Beyond its conceptual simplicity, the approach operates in continuous spacetime, supports an explicit implementation of chiral symmetry, and includes a resummation of beyond-ladder diagrams already in simple truncations. Moreover, we monitor gauge invariance with the aid of Ward-Takahashi identities and study regulator-scheme dependencies. Since the critical number of flavors is a universal quantity, the scheme dependencies are a direct measure of the approximation uncertainties of our non-perturbative truncation. Apart from a quantitative prediction for $N_{\mathrm{f}}^{\text {cr }}$, our results reveal further facets of the nature of the phase transition. Across the phase transition, we particularly observe discontinuities in the effective fermionic self-interactions which are generated by the RG flow.

For our analysis, we employ the functional RG formulated in terms of a flow equation for the effective average action $\Gamma_{k}[10]$,

$$
\partial_{t} \Gamma_{k}=\frac{1}{2} \mathrm{~S} \operatorname{Tr} \partial_{t} R_{k}\left(\Gamma_{k}^{(2)}+R_{k}\right)^{-1}, \quad t=\ln \frac{k}{\Lambda_{\mathrm{UV}}},
$$

serving as an alternative definition of the quantum field theory. $\Gamma_{k}$ is a free-energy functional that interpolates between the bare action $\Gamma_{k=\Lambda_{\mathrm{UV}}}=S$ and the full quantum effective action $\Gamma=\Gamma_{k=0}$. Here, $R_{k}$ denotes a regulator function that specifies the details of the momentum-shell integrations, the variation of which will provide us with an error estimate of our approximations. We devote Sect. 2 to a discussion of our approximation scheme, which represents a truncation of the full theory to the relevant operators for the present problem. Results and conclusions are presented in Sects. 3 and 4.

\section{Flow equations}

Our primary aim is a reliable determination of the critical flavor number $N_{\mathrm{f}}^{\text {cr }}$. For this, the approach of the phase transition from the symmetric side is technically advantageous, since the relevant degrees of freedom are expected to remain the same during the flow from the ultraviolet (UV) to the infrared (IR): quarks and gluons. Therefore, we solve the flow equation (1) in a truncated subspace of all possible action functionals.

In addition to standard gauge and fermion sectors, we include all possible pointlike four-fermion interactions that are compatible with $\mathrm{SU}\left(N_{\mathrm{c}}\right)$ gauge symmetry and a chiral SU $\left(N_{\mathrm{f}}\right)_{\mathrm{L}} \times \mathrm{SU}\left(N_{\mathrm{f}}\right)_{\mathrm{R}}$ flavor symmetry for $N_{\mathrm{f}}$ fermion species $^{2}$,

$$
\begin{aligned}
\Gamma_{k}=\int & \bar{\psi}\left(\mathrm{i} Z_{\psi} \not \partial+Z_{1} \bar{g} \not A\right) \psi+\frac{Z_{A}}{4} F_{z}^{\mu \nu} F_{\mu \nu}^{z}+\frac{\left(\partial_{\mu} A^{\mu}\right)^{2}}{2 \alpha} \\
+ & \frac{1}{2}\left[Z_{-} \bar{\lambda}_{-}(\mathrm{V}-\mathrm{A})+Z_{+} \bar{\lambda}_{+}(\mathrm{V}+\mathrm{A})+Z_{\sigma} \bar{\lambda}_{\sigma}(\mathrm{S}-\mathrm{P})\right. \\
& \left.+Z_{\mathrm{VA}} \bar{\lambda}_{\mathrm{VA}}\left[2(\mathrm{~V}-\mathrm{A})^{\mathrm{adj}}+\left(1 / N_{\mathrm{c}}\right)(\mathrm{V}-\mathrm{A})\right]\right] \mathrm{d}^{4} x
\end{aligned}
$$

Here $A_{\mu}=A^{z} T^{z}, F_{\mu \nu}=F_{\mu \nu}^{z} T^{z}$ denote the nonabelian gauge potential and field strength (the inclusion of a ghost sector is tacitly assumed). The gauge-field kinetic term is accompanied by a $k$-dependent wavefunction renormalization $Z_{A}$, the fermionic one by $Z_{\psi}$. Similarly, $Z_{1}, Z_{+}, Z_{-}$, $Z_{\sigma}$ and $Z_{\mathrm{VA}}$ are the vertex renormalizations, whereas $\bar{g}$, $\bar{\lambda}$ denote the bare couplings. Renormalized dimensionless couplings are defined by

$$
g=\frac{\bar{g} Z_{1}}{Z_{A}^{1 / 2} Z_{\psi}}, \quad \lambda_{i}=\frac{Z_{i} k^{2} \overline{\lambda_{i}}}{Z_{\psi}^{2}} .
$$

We work in the Landau gauge, $\alpha=0$, which is known to be a fixed point of the renormalization group [12] and has the additional advantage that the fermionic wavefunction is not renormalized in our truncation, so that we can choose $Z_{\psi}=1$.

The four-fermion interactions can be classified according to their color and flavor structure. Color and flavor singlets are

$$
\begin{aligned}
(\mathrm{V}-\mathrm{A}) & =\left(\bar{\psi} \gamma_{\mu} \psi\right)^{2}+\left(\bar{\psi} \gamma_{\mu} \gamma_{5} \psi\right)^{2}, \\
(\mathrm{~V}+\mathrm{A}) & =\left(\bar{\psi} \gamma_{\mu} \psi\right)^{2}-\left(\bar{\psi} \gamma_{\mu} \gamma_{5} \psi\right)^{2},
\end{aligned}
$$

where color $(i, j, \ldots)$ and flavor $(a, b, \ldots)$ indices are contracted pairwise, e.g., $(\bar{\psi} \psi) \equiv\left(\bar{\psi}_{i}^{a} \psi_{i}^{a}\right)$. The remaining operators have non-trivial color or flavor structure,

$$
\begin{aligned}
(\mathrm{S}-\mathrm{P}) & =\left(\bar{\psi}^{a} \psi^{b}\right)^{2}-\left(\bar{\psi}^{a} \gamma_{5} \psi^{b}\right)^{2} \\
& \equiv\left(\bar{\psi}_{i}^{a} \psi_{i}^{b}\right)^{2}-\left(\bar{\psi}_{i}^{a} \gamma_{5} \psi_{i}^{b}\right)^{2}, \\
(\mathrm{~V}-\mathrm{A})^{\mathrm{adj}} & =\left(\bar{\psi} \gamma_{\mu} T^{z} \psi\right)^{2}+\left(\bar{\psi} \gamma_{\mu} \gamma_{5} T^{z} \psi\right)^{2},
\end{aligned}
$$

where $\left(\bar{\psi}^{a} \psi^{b}\right)^{2} \equiv \bar{\psi}^{a} \psi^{b} \bar{\psi}^{b} \psi^{a}$, etc., and $\left(T^{z}\right)_{i j}$ denotes the generators of the gauge group in the fundamental representation. The set of fermionic self-interactions occurring in (2) forms a complete basis. Any other pointlike fourfermion interaction invariant under $\mathrm{SU}\left(N_{\mathrm{c}}\right)$ gauge symmetry and $\mathrm{SU}\left(N_{\mathrm{f}}\right)_{\mathrm{L}} \times \mathrm{SU}\left(N_{\mathrm{f}}\right)_{\mathrm{R}}$ flavor symmetry is reducible by means of Fierz transformations.

It is important to stress that the effective action (2) is in the QCD universality class [13], as long as the fourfermion interactions are small in the UV and thus RG irrelevant. Only non-perturbatively large $\left(Z_{i} \bar{\lambda}_{i}\right)$ 's would bring

2 We note that only the four-fermion interactions are manifestly invariant under local gauge transformations for all possible choices of the couplings. Gauge invariance of the remaining terms is governed by (modified) Ward-Takahashi identities as discussed for the present system in [11]. 
us into another universality class with $\chi \mathrm{SB}$ of NambuJona-Lasinio (NJL) type, which will not be considered in this work.

Plugging the truncated effective action (2) into the flow equation, we obtain the following $\beta$ functions for the dimensionless couplings $\lambda_{i}[11]$ :

$$
\begin{aligned}
& \partial_{t} \lambda_{-}= 2 \lambda_{-}-4 v_{4} l_{1,1}^{(\mathrm{FB}), 4}\left[\frac{3}{N_{\mathrm{c}}} g^{2} \lambda_{-}-3 g^{2} \lambda_{\mathrm{VA}}\right] \\
&-\frac{1}{8} v_{4} l_{1,2}^{(\mathrm{FB}), 4}\left[\frac{12+9 N_{\mathrm{c}}^{2}}{N_{\mathrm{c}}^{2}} g^{4}\right] \\
&-8 v_{4} l_{1}^{(\mathrm{F}), 4}\left\{-N_{\mathrm{f}} N_{\mathrm{c}}\left(\lambda_{-}^{2}+\lambda_{+}^{2}\right)+\lambda_{-}^{2}\right. \\
&\left.-2\left(N_{\mathrm{c}}+N_{\mathrm{f}}\right) \lambda_{-} \lambda_{\mathrm{VA}}+N_{\mathrm{f}} \lambda_{+} \lambda_{\sigma}+2 \lambda_{\mathrm{VA}}^{2}\right\} \\
& \partial_{t} \lambda_{+}= 2 \lambda_{+}-4 v_{4} l_{1,1}^{(\mathrm{FB}), 4}\left[-\frac{3}{N_{\mathrm{c}}} g^{2} \lambda_{+}\right] \\
&-\frac{1}{8} v_{4} l_{1,2}^{(\mathrm{FB}), 4}\left[-\frac{12+3 N_{\mathrm{c}}^{2}}{N_{\mathrm{c}}^{2}} g^{4}\right] \\
&-8 v_{4} l_{1}^{(\mathrm{F}), 4}\left\{-3 \lambda_{+}^{2}-2 N_{\mathrm{c}} N_{\mathrm{f}} \lambda_{-} \lambda_{+}\right. \\
&-2 \lambda_{+}\left(\lambda_{-}+\left(N_{\mathrm{c}}+N_{\mathrm{f}}\right) \lambda_{\mathrm{VA}}\right)+N_{\mathrm{f}} \lambda_{-} \lambda_{\sigma} \\
&\left.+\lambda_{\mathrm{VA}} \lambda_{\sigma}+\frac{1}{4} \lambda_{\sigma}^{2}\right\} \\
& \partial_{t} \lambda_{\sigma}= 2 \lambda_{\sigma}-4 v_{4} l_{1,1}^{(\mathrm{FB}), 4}\left[6 C_{2}\left(N_{\mathrm{c}}\right) g^{2} \lambda_{\sigma}-6 g^{2} \lambda_{+}\right] \\
&-\frac{1}{4} v_{4} l_{1,2}^{(\mathrm{FB}), 4}\left[-\frac{24-9 N_{\mathrm{c}}^{2}}{N_{\mathrm{c}}} g^{4}\right] \\
&-8 v_{4} l_{1}^{(\mathrm{F}), 4}\left\{2 N_{\mathrm{c}} \lambda_{\sigma}^{2}-2 \lambda_{-} \lambda_{\sigma}\right. \\
&\left.-2 N_{\mathrm{f}} \lambda_{\sigma} \lambda_{\mathrm{VA}}-6 \lambda_{+} \lambda_{\sigma}\right\} \\
& \partial_{t} \lambda_{\mathrm{VA}}=2 \lambda_{\mathrm{VA}}-4 v_{4} l_{1,1}^{(\mathrm{FB}), 4}\left[\frac{3}{N_{\mathrm{c}}} g^{2} \lambda_{\mathrm{VA}}-3 g^{2} \lambda_{-}\right] \\
&- \frac{1}{8} v_{4} l_{1,2}^{(\mathrm{FB}), 4}\left[-\frac{24-3 N_{\mathrm{c}}^{2}}{N_{\mathrm{c}}} g^{4}\right] \\
&-8 v_{4} l_{1}^{\mathrm{F}), 4}\left\{-\left(N_{\mathrm{c}}+N_{\mathrm{f}}\right) \lambda_{\mathrm{VA}}^{2}+4 \lambda_{-} \lambda_{\mathrm{VA}}-\frac{1}{4} N_{\mathrm{f}} \lambda_{\sigma}^{2}\right\} \\
&
\end{aligned}
$$

Here $C_{2}\left(N_{\mathrm{c}}\right)=\left(N_{\mathrm{c}}^{2}-1\right) /\left(2 N_{\mathrm{c}}\right)$ is a Casimir operator of the gauge group, and $v_{4}=1 /\left(32 \pi^{2}\right)$. For better readability, we have written all gauge-coupling-dependent terms in square brackets, whereas fermionic self-interactions are grouped inside braces. The threshold functions $l$ characterize the regulator dependence [14]. For the "optimized" linear regulator [15], these read

$$
l_{1}^{(\mathrm{F}), 4}=\frac{1}{2}, \quad l_{1,1}^{(\mathrm{FB}), 4}=1-\frac{\eta_{\mathrm{A}}}{6}, \quad l_{1,2}^{(\mathrm{FB}), 4}=\frac{3}{2}-\frac{\eta_{A}}{6} .
$$

Next, let us turn to the running of the gauge coupling. Even though a non-perturbative estimate can already be obtained within the current simple truncation [16], a reliable estimate to higher loops requires the inclusion of many further vertex operators in the truncation (2). Instead, we construct the $\beta$ function according to the following line of argument: First, we note that gauge invariance in the RG scheme can be monitored with the aid of regulator-dependent Ward-Takahashi identities [16, 17].
For the present system, these identities constrain the fourfermion contributions to the running gauge coupling to be of the form [11]

$$
\begin{aligned}
& \partial_{t} g^{2}=\breve{\eta}_{g^{2}} g^{2}-4 v_{4} l_{1}^{(\mathrm{F}), 4} \frac{g^{2}}{1-2 v_{4} l_{1}^{(\mathrm{F}), 4} \sum c_{i} \lambda_{i}} \partial_{t} \sum c_{i} \lambda_{i}, \\
& c_{\sigma}=1+N_{\mathrm{f}}, \quad c_{+}=0, c_{-}=-2, c_{\mathrm{VA}}=-2 N_{\mathrm{f}},
\end{aligned}
$$

where

$$
\begin{aligned}
\breve{\eta}_{g^{2}} & =\eta_{\mathrm{A}}-2 \breve{\eta}_{1}+2 \eta_{\psi} \\
& =-4 v_{4} g^{2}\left(\beta_{0}+\beta_{1}\left(2 v_{4} g^{2}\right)+\beta_{2}\left(2 v_{4} g^{2}\right)^{2}+\ldots\right)
\end{aligned}
$$

is the standard gauge-coupling anomalous dimension, trivially related to the standard $\beta$ function by $\breve{\beta}_{g}^{2}=\breve{\eta}_{g^{2}} g^{2} \cdot \eta_{\mathrm{A}}$, $\eta_{\psi}$ and $\eta_{1}$ are the gluon, quark and quark-gluon-vertex anomalous dimensions, respectively, defined by $\eta_{i}=-\partial_{t}$ $\ln Z_{i} ; \eta_{\psi}$, in fact, vanishes in the present truncation using the Landau gauge. The symbol denotes those standard contributions which are not generated by the modified Ward-Takahashi identities; for instance, the difference between $\eta_{1}$ and $\breve{\eta}_{1}$ is exactly given by the term $\sim \partial_{t} \lambda_{i}$ in (13).

Instead of computing $\breve{\eta}_{g^{2}}$ in the RG scheme, we use the four-loop result obtained in the $\overline{\mathrm{MS}}$ scheme $[18,19]$ as an estimate. For a qualitative discussion, let us list the first two coefficients (all four known coefficients are used in the numerics),

$$
\beta_{0}=\frac{11}{3} N_{\mathrm{c}}-\frac{2}{3} N_{\mathrm{f}}, \beta_{1}=\frac{34 N_{\mathrm{c}}^{3}+3 N_{\mathrm{f}}-13 N_{\mathrm{c}}^{2} N_{\mathrm{f}}}{3 N_{\mathrm{c}}},
$$

with $\beta_{1}$ reversing its sign for $N_{\mathrm{f}}>\frac{34 N_{\mathrm{c}}^{3}}{13 N_{\mathrm{c}}^{2}-3}$. Since we do not expect that the RG- and $\overline{\mathrm{MS}}$-scheme $\beta$ functions exactly coincide, this four-loop approximation introduces an error that is estimated below. ${ }^{3}$ It should be stressed at this point that some crucial properties of the $\beta$ function are not scheme dependent; in particular, the existence of zeros, i.e., fixed points, is a universal result which is relevant for the present problem.

One further technical point needs to be mentioned: whereas the running gauge coupling is dominated by $\breve{\eta}_{g^{2}}$, the threshold functions $l$ of the fermionic sector require the separate knowledge of $\eta_{\mathrm{A}}$. As a rough but sufficient estimate, we simply employ the one-loop relation for the Landau gauge, $\eta_{\mathrm{A}}^{1-\text { loop }}=\frac{13}{22} \breve{\eta}_{g^{2}}^{1 \text {-loop }}$. Since the anomalous dimensions turn out to remain numerically small of $\mathcal{O}(0.1)$ and the threshold functions only depend on $\frac{\eta_{\mathrm{A}}}{6}$, this additional higher-order approximation has little effect on the result. ${ }^{4}$

3 Note that the additional terms $\sim \partial_{t} \lambda_{i}$ in (13) already contribute to the resulting $\beta$ function at two-loop order. By approximating $\breve{\eta}_{g^{2}}$ by the $\overline{\mathrm{MS}}$ result, we introduce a potential double counting of diagrams at this and higher order. However, the $\partial_{t} \lambda_{i}$ contributions vanish at the fixed point. In addition, we would like to stress that we are using a mass-dependent regularization scheme, and universality of the second coefficient in the $\beta$ function does not hold and further contributions at this order are expected.

4 As a check, we have used the pure one-loop result $\eta_{\mathrm{A}}^{1-\text { loop }}$ which is non-vanishing at the fixed point. The corresponding results lie well within the estimated error (shaded region in Fig. 3). 


\section{Results}

The onset of $\chi \mathrm{SB}$ is detected by the four-fermion part of our model (2). Chiral symmetry remains preserved in the pointlike limit, as long as the fermion self-interactions remain finite. In turn, $\chi \mathrm{SB}$ is signaled by a diverging $\lambda_{i}$. This seeming Landau-pole behavior points to the formation of chiral condensates, since the $\lambda_{i}$ 's are inversely proportional to the mass parameter of a Ginzburg-Landau potential for the order parameter in a (partially) bosonized language, $\lambda_{i} \sim 1 / m_{i}^{2}$.

Therefore, let us first consider the $\lambda_{i}$ flow separately. For vanishing gauge coupling, the flow is solved by vanishing $\lambda_{i}$ 's, which is the Gaussian fixed point. This fixed point is IR attractive, implying that these self-interactions are RG irrelevant for sufficiently small bare couplings, as they should be. Since the $\lambda_{i}$ flows are quadratic in the $\lambda_{i}$ 's corresponding to a parabola, there are 15 further non-Gaußian fixed points $\left(2^{\left(\# \lambda_{i}=4\right)}=16\right.$ in total). These are related to the set of critical couplings $\lambda_{i, \mathrm{cr}}$ beyond which the system is in the universality class of NJL type; see Fig. 1.

For fixed small gauge coupling, the Gaußian fixed point is shifted a bit to fixed-point values of the order $\lambda_{*, i} \sim g^{4}$, effectively describing scattering of massless quarks. This fixed point is still IR attractive, see Fig. 1; hence there is no $\chi \mathrm{SB}$ as long as the gauge coupling remains small enough.

If the gauge coupling becomes larger than a critical value $\alpha_{\mathrm{cr}}=g_{\mathrm{cr}}^{2} / 4 \pi$, all fixed points of the $\lambda_{i}$ flows are removed, so that the $\lambda_{i}$ 's quickly run into a divergence, signaling $\chi \mathrm{SB}[20]$.

In Fig. 2, we study the four-fermion system at fixed gauge coupling. For various numbers of colors $N_{\mathrm{c}}$, we plot the critical gauge coupling $\alpha_{\mathrm{cr}}=g_{\mathrm{cr}}^{2} / 4 \pi$ at which the fermion system becomes critical and exhibits $\chi \mathrm{SB}$. For an increasing number of colors, $\alpha_{\text {cr }}$ decreases. The dependence on the number of flavors is rather weak.

Now, let us switch on the running of the gauge coupling. The above-mentioned fixed points in the fermionic system become quasi-fixed points which persist for sufficiently weak gauge coupling; their values are modulated by

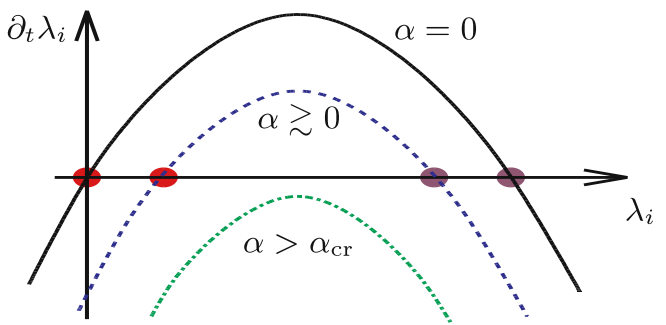

Fig. 1. Sketch of a typical $\beta$ function for the fermionic selfinteractions $\lambda_{i}$ : at zero gauge coupling, $\alpha=0$ (solid curve), the Gaußian fixed point $\lambda_{i}=0$ is IR attractive (the second fixed point at $\lambda_{i}>0$ corresponds to the IR repulsive critical coupling of NJL type). For small $\alpha \gtrsim 0$ (dashed curve), the fixed-point positions are shifted on the order of $\alpha^{2}$. For gauge couplings larger than the critical coupling $\alpha>\alpha_{\mathrm{cr}}$ (dot-dashed curve), no fixed points remain and the self-interactions quickly grow large, signaling $\chi \mathrm{SB}$. We emphasize that both fixed-points values remain finite until the fixed points eventually vanish at $\alpha_{\mathrm{cr}}$

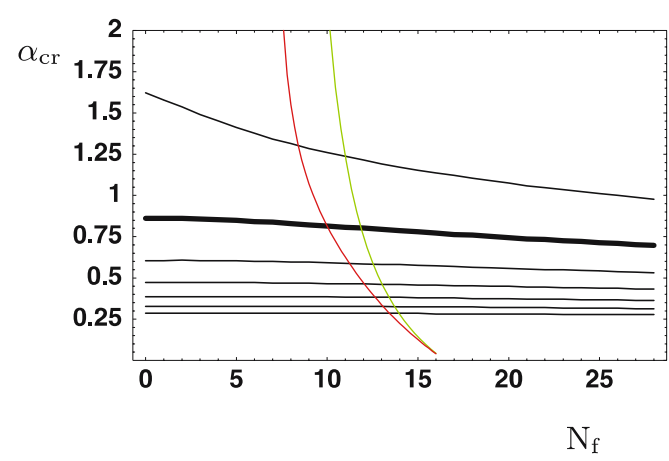

Fig. 2. Critical coupling for the four-fermion system. From top to bottom, the number of colors increases from $N_{\mathrm{c}}=2$ to $N_{\mathrm{c}}=8$ (The thick line corresponds to $N_{\mathrm{c}}=3$ ). The red/darkgrey (green/light-grey) line shows the fixed-point gauge coupling for $N_{\mathrm{c}}=3$ at four (two) loop. At the crossing, the critical number of flavors can be read off

the logarithmic increase of the gauge coupling. If the gauge coupling grows larger than the critical value, the fermionic quasi-fixed points vanish (the parabola drops below the $\lambda_{i}$ axis in Fig. 1), and the system runs into the $\chi \mathrm{SB}$ regime. In this way, the IR physics is solely determined by the gauge coupling, as expected. Whether or not the system ends up in the $\chi \mathrm{SB}$ regime finally depends on the maximal value of the gauge coupling. For a large number of flavors, $N_{\mathrm{f}} \lesssim N_{\mathrm{f}}^{\text {a.f. }}$, this value is given by the IR fixed-point value $\alpha_{*}$, induced by the higher $\beta$ function coefficients. Lowering $N_{\mathrm{f}}$ increases $\alpha_{*}$, eventually exceeding $\alpha_{\mathrm{cr}}$ required for $\chi \mathrm{SB}$.

We determine the resulting critical number of flavors $N_{\mathrm{f}}^{\text {cr }}$ by comparing the fixed point of the gauge coupling, $\alpha_{*} \equiv \alpha_{*}\left(N_{\mathrm{f}}\right)$, to $\alpha_{\mathrm{cr}}$. The task of solving the complete coupled set of fixed-point equations $\partial_{t} g^{2}=0, \partial_{t} \lambda=0$ becomes simplified by the following observation: (13) reveals that the only contributions to $\partial_{t} g^{2}$ involving $\lambda$ are proportional $\partial_{t} \lambda$. By definition this term vanishes at any fixed point in the subsystem of four-fermion interactions. Therefore, the gauge coupling at any fixed point of the complete system is determined by $\breve{\eta}_{g^{2}}=0$, which depends only on $g$. In Fig. 2, the fixed-point gauge coupling $\alpha_{*}\left(N_{\mathrm{f}}\right)$ is plotted for $N_{\mathrm{c}}=3$ with a two- (green/light-grey) and a four-loop (red/dark-grey) result for $\breve{\eta}_{g^{2}}$.

The critical number of flavors $N_{f}^{\text {cr }}$ can now be read off from the intersection between this line and the critical coupling $\alpha_{\text {cr }}$ of the four-fermion subsystem for $N_{\mathrm{c}}=3$ (thick solid line). In the same way, we determine the critical number of flavors as a function of $N_{\mathrm{c}}$, as plotted in Fig. $3 .^{5}$

The regulator dependence of the flow equation offers a possibility for estimating the error of our quantitative results which is introduced by the truncation. Exact results for physical quantities like $N_{\mathrm{f}}^{\text {cr }}$ are universal without any regulator dependence. However, this universality

\footnotetext{
5 An equivalent way to determine $N_{\mathrm{f}}^{\mathrm{cr}}\left(N_{\mathrm{c}}\right)$ is to directly solve the flow equations (8)-(13) with $\lambda_{i}(t=0)=0$ and a small value $g^{2}(t=0)$ as initial conditions. One can then find $N_{\mathrm{f}}^{\text {cr }}$ by looking for the infimum of all $N_{\mathrm{f}}$ for which the four-fermion couplings do not diverge at a finite $t$, (or alternatively, for the supremum of those which do diverge).
} 


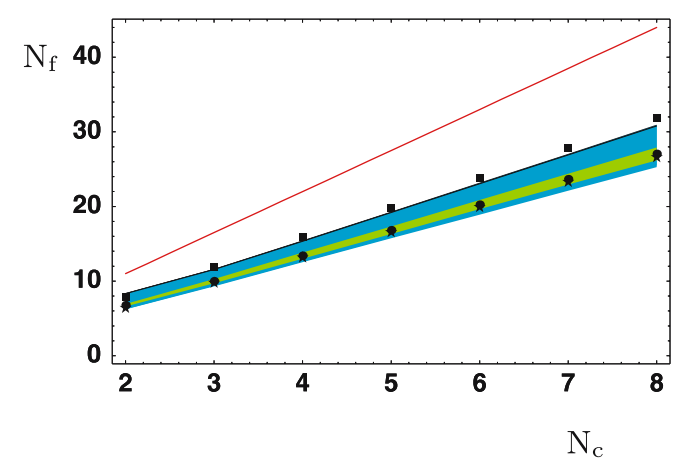

Fig. 3. Critical number of flavors for $\mathrm{SU}\left(N_{\mathrm{c}}\right)$ gauge theory. The result based on the four-loop $\overline{\mathrm{MS}}$ beta function is denoted by black circles which lie almost on top of stars, representing the three-loop result; black boxes correspond to the twoloop beta function. The (inner) green/dark-grey shaded region around the four-loop result displays our error estimate for the fermionic sector. The (outer) turquois/light-grey shaded region shows the approximate gluonic error, estimated by a variation of the higher-loop coefficients. The red/grey line shows the number of flavors above which asymptotic freedom is lost

does not hold for the truncated flow, so that the regulator dependence of would-be universal quantities directly translates into an error of the truncation. Let us start with the fermionic subsystem, for which the pointlike approximation of vertices and the use of "classical" propagators represents the most severe approximation. If these truncated non-trivial momentum dependencies of vertices and propagators were important for the determination of the universal $N_{f}$, we would expect a strong regulator dependence within the pointlike approximation. This is because the flow equation is localized in momentum space and different regulators probe the vertices and propagators at different momentum shells; consequently, erroneously truncated momentum dependencies of the vertices generically imply strong variations of would-be universal quantities for different regulators. In this sense, the study of regulator dependencies is an important if not crucial test of our pointlike approximation in the fermion sector for the determination of $N_{f}$. In the present case, the regulator dependence occurs in the threshold functions $l$. Our pointlike truncation is reminiscent of a derivative expansion, for which a flow-optimization procedure has led to the construction of a linear regulator [15] used in (12). Following the stability arguments of [15], we consider the results from this regulator as our "optimized" predictions. The opposite limit of non-optimized regulators for pointlike interactions is marked by the sharp cutoff $[21]$ with $l_{1}^{(\mathrm{F}), 4}=l_{1,1}^{(\mathrm{FB}), 4}=$ $l_{1,2}^{(\mathrm{FB}), 4}=1$, neglecting the anomalous dimension. Therefore, a conservative error estimate is given by the difference between the sharp cutoff and the optimized regulator. ${ }^{6}$ This results in the green/dark-grey shaded region in Fig. 3. The resulting error is actually very small; in particular, we

\footnotetext{
6 The sharp cutoff can be considered as the limit of a sequence of powerlike regulators, the results of which all lie within the estimated error.
}

observe strong non-trivial cancellations between the contributions of different threshold functions to the final result. Since this exactly matches with the expectation for universal quantities, it provides evidence for the reliability of the pointlike truncation for the present purpose. As a further evidence for the consistency of the pointlike approximation in the symmetric phase, we note that the gluon as well as the quark anomalous dimension vanish at the IR fixed point in our approximation, implying that the use of "classical" propagators is self-consistent here. Of course, we should stress that the pointlike approximation is expected to break down for many other questions, in particular, those related to the properties of the $\chi \mathrm{SBphase}$.

The largest uncertainty arises from the gauge sector. As an error estimate, one might use the difference between the various loop orders in the $\beta$-function contribution $\breve{\eta}_{q^{2}}$. As depicted in Fig. 3, the difference between two and three loops in the $\overline{\mathrm{MS}}$ scheme is of the order $\Delta N_{\mathrm{f}}^{\text {cr }} \sim 1$. The difference between the three- and the four-loop result is already much smaller. This suggests that, for our purposes, the gauge sector is reliably approximated by the four-loop $\beta$ function. However, as discussed above, the (known) $\overline{\mathrm{MS}}$ result is not expected to coincide exactly with the (unknown) result to be obtained with the linear regulator used so far. For a quantitative estimate of the resulting error, we can perform a comparison at two-loop level, where the massdependent character of the flow equation regularization already introduces scheme dependencies. On a quantitative level, this introduces differences on the $10 \%$ level [22]. ${ }^{7}$ For the three- and four-loop coefficients we assume larger uncertainties on the $30 \%$ and $50 \%$ level, respectively. This results in an error depicted by the turquois/light-grey shaded region in Fig. 3. Quadratically adding both uncertainties, the resulting error is dominated by the gluonic sector.

It has been argued [24] that a regularization scheme exists in which the two-loop $\beta$ function for the gauge coupling is exact. We stress that this does not imply that the twoloop curve in Fig. 3 is exact, since this particular unknown regularization scheme may involve a regulator with strong quantitative influence on the fermionic sector (possibly requiring operators beyond the pointlike limit). Hence, it is well possible that the resulting curve of that special scheme is again close to the estimated four-loop result in our scheme.

It is worth pointing out that the total estimated error is significantly smaller than the distance to the line $N_{\mathrm{f}}^{\text {a.f. }}\left(N_{\mathrm{c}}\right)$ where asymptotic freedom is lost. This strongly confirms the existence of a phase of many-flavor QCD which is asymptotically free, but has no $\chi$ SBand no strict confinement in the IR.

\section{Summary and conclusions}

We have used flow equations to determine the critical number of flavors $N_{\mathrm{f}}^{\text {cr }}$ separating the phases with and without

\footnotetext{
7 For an exact computation of the universal two-loop coefficient within the functional RG approach, see [23].
} 
$\chi \mathrm{SB}$. Our truncation includes all pointlike four-fermion interactions allowed by gauge and chiral symmetry which are generated by ladder as well as non-ladder processes; thereby, our result represents a significant improvement over conventional approximation techniques. Gauge symmetry is monitored by modified Ward-Takahashi identities, resulting in an additional four-fermion contribution to the flow of the gauge coupling. We have demonstrated that these terms have no effect on $N_{\mathrm{f}}^{\text {cr }}$, owing to the structure of the modified Ward-Takahashi identity (by contrast, these terms are of quantitative importance, for instance, for the Landau-pole problem of QED [25]).

Our findings confirm the existence of a sizable parameter region $N_{\mathrm{f}}^{\text {cr }}<N_{\mathrm{f}}<N_{\mathrm{f}}^{\text {a.f. }}$ where chiral symmetry remains unbroken, in agreement with earlier results [2-6]. Our result for the critical number of flavors, for instance, in $\mathrm{SU}(3)$ gauge theory, is $N_{\mathrm{f}}^{\text {cr }}=10.0 \pm 0.29$ (fermion) ${ }_{-0.63}^{+1.55}$ (gluon). The errors result from the fermionic and gluonic sectors of our truncation, respectively, the quantitative influence of which we have estimated from the regulator dependence of universal quantities.

Our restriction to a truncation in terms of quark and gluon fields with pointlike interaction does not facilitate a study of the $\chi \mathrm{SB}$ regime; for this, the inclusion of bosonic composite fields along the lines of $[13,20,26,27]$ represents a powerful tool.

Nevertheless, important aspects of the unusual nature of the phase transition can already be read off from the fermionic sector: as long as the system is in the symmetric phase, $N_{\mathrm{f}}^{\text {cr }}<N_{\mathrm{f}}<N_{\mathrm{f}}^{\text {a.f. }}$, the fermionic self-interactions approach fixed-point values in the IR which are finite numbers. Even arbitrarily close to the phase transition, these numbers remain finite and are elements of a compact region in parameter space, $\left.\lambda_{i}\right|_{k \rightarrow 0} \in \Omega_{\lambda}$. By contrast, the self-interactions diverge in the broken phase where all fixed points have vanished. As a consequence, an infinite region in parameter space outside $\Omega_{\lambda}$ remains inaccessible. Varying $N_{\mathrm{f}}$ continuously across the phase transition coming from the symmetric side, the fermionic selfinteractions jump discontinuously at $N_{\mathrm{f}}=N_{\mathrm{f}}^{\text {cr }}$. Now, the $\lambda_{i}$ 's are inversely proportional to the mass parameter of a Ginzburg-Landau potential for the order parameter in a (partially) bosonized language, $\lambda_{i} \sim 1 / m_{i}^{2}$; hence, our observation in the fermionic sector agrees with the observations of $[2,3,9]$ that the phase transition is not conventionally second order, even though the chiral order parameter is known to change continuously across the chiral phase transition [13]. Our work thus reveals further aspects of the nature of this type of zero-temperature phase transitions which may find further application in related systems such as $\mathrm{QED}_{3}$ or models of electroweak symmetry breaking.

Acknowledgements. J.J. would like to thank the ITP in Heidelberg for its hospitality and A. Ringwald for useful comments. H.G. is grateful to J.M. Pawlowski for discussions and acknowl- edges support by the Deutsche Forschungsgemeinschaft (DFG) under contract Gi 328/1-3 (Emmy-Noether program).

\section{References}

1. T. Banks, A. Zaks, Nucl. Phys. B 196, 189 (1982)

2. V.A. Miransky, K. Yamawaki, Phys. Rev. D 55, 5051 (1997); Erratum-ibid. D 56, 3768 (1997) [hep-th/9611142]

3. T. Appelquist, J. Terning, L.C.R. Wijewardhana, Phys. Rev. Lett. 77, 1214 (1996) [hep-ph/9602385]; T. Appelquist, A. Ratnaweera, J. Terning, L.C.R. Wijewardhana, Phys. Rev. D 58, 105017 (1998) [hep-ph/9806472]

4. M. Harada, M. Kurachi, K. Yamawaki, Phys. Rev. D 68, 076001 (2003) [hep-ph/0305018]

5. Y. Iwasaki, K. Kanaya, S. Kaya, S. Sakai, T. Yoshie, Phys. Rev. D 69, 014507 (2004) [hep-lat/0309159]

6. F. Sannino, J. Schechter, Phys. Rev. D 60, 056004 (1999) [hep-ph/9903359]

7. T. Appelquist, S.B. Selipsky, Phys. Lett. B 400, 364 (1997) [hep-ph/9702404]; M. Velkovsky, E.V. Shuryak, Phys. Lett. B 437, 398 (1998) [hep-ph/9703345]

8. M. Harada, K. Yamawaki, Phys. Rev. Lett. 86, 757 (2001) [hep-ph/0010207]

9. R.S. Chivukula, Phys. Rev. D 55, 5238 (1997) [hep$\mathrm{ph} / 9612267$

10. C. Wetterich, Phys. Lett. B 301, 90 (1993); Nucl. Phys. B 352, 529 (1991); Z. Phys. C 48, 693 (1990)

11. H. Gies, J. Jaeckel, C. Wetterich, Phys. Rev. D 69, 105008 (2004) [hep-ph/0312034]

12. U. Ellwanger, M. Hirsch, A. Weber, Z. Phys. C 69, 687 (1996) [hep-th/9506019]; D.F. Litim, J.M. Pawlowski, Phys. Lett. B 435, 181 (1998) [hep-th/9802064]

13. H. Gies, C. Wetterich, Phys. Rev. D 69, 025001 (2004) [hep-th/0209183]

14. D.U. Jungnickel, C. Wetterich, Phys. Rev. D 53, 5142 (1996) [hep-ph/9505267]

15. D.F. Litim, Phys. Lett. B 486, 92 (2000) [hep-th/0005245]; Phys. Rev. D 64, 105007 (2001) [hep-th/0103195]

16. M. Reuter, C. Wetterich, Nucl. Phys. B 417, 181 (1994)

17. U. Ellwanger, Phys. Lett. B 335, 364 (1994) [hep-th/ 9402077]; F. Freire, D.F. Litim, J.M. Pawlowski, Phys. Lett. B 495, 256 (2000) [hep-th/0009110]

18. T. van Ritbergen, J.A.M. Vermaseren, S.A. Larin, Phys. Lett. B 400, 379 (1997) [hep-ph/9701390]

19. M. Czakon, Nucl. Phys. B 710, 485 (2005) [hep-ph/0411261]

20. H. Gies, C. Wetterich, Phys. Rev. D 65, 065001 (2002) [hep-th/0107221]

21. D.F. Litim, Nucl. Phys. B 631, 128 (2002) [hep-th/0203006]

22. M. Reuter, C. Wetterich, Phys. Rev. D 56, 7893 (1997) [hep-th/9708051]; H. Gies, Phys. Rev. D 66, 025006 (2002) [hep-th/0202207]

23. J.M. Pawlowski, Int. J. Mod. Phys. A 16, 2105 (2001)

24. G. 't Hooft, in: NATO Advanced Study Inst., Ser. B, v.59, ed. by G. t Hooft et al. (Plenum Press, New York, 1980)

25. H. Gies, J. Jaeckel, Phys. Rev. Lett. 93, 110405 (2004) [hep-ph/0405183]

26. J. Jaeckel, C. Wetterich, Phys. Rev. D 68, 025020 (2003) [hep-ph/0207094]

27. J. Jaeckel, hep-ph/0309090 